\title{
Petites choses et petites gens dans Le Cousin Pons
}

Thomas Conrad

(ENS Paris, PSL)

En lisant Le Cousin Pons, nous sommes amenés à nous prononcer sur l'évolution de Balzac dans les dernières années de sa création. L'interpréterons-nous comme un épuisement, comme un approfondissement, comme un renouvellement?

a) Du côté de l'épuisement, on relèvera l'abattement physique et moral de l'auteur, la noirceur d'un univers dominé par la mort et la cruauté, l'hostilité affichée vis-à-vis du présent médiocre de la monarchie de Juillet : on esquisse alors un Cousin Pons sombre, mélancolique, avec ses nuances de sarcasme, d'amertume et de cynisme.

b) Du côté de l'approfondissement, on verra Le Cousin Pons comme le prolongement logique de l'histoire des mœurs commencée depuis les années 1830. La cohérence du projet s'affirme par le rattachement obstiné des derniers romans à La Comédie bumaine (retour des personnages, inscription dans les Scènes de la vie parisienne dans un tome supplémentaire du Furne, variations sur les motifs d'autres romans $\left.{ }^{1}\right)$. On montrerait la permanence des grands thèmes balzaciens : la passion, l'énergie vitale, l'indifférenciation de la société bourgeoise, le triomphe de l'argent, etc. Ce serait un Cousin Pons de part en part «balzacien », écrit, envers et contre tout, par un Balzac persévérant dans la singularité de son projet créateur.

c) Du côté du renouvellement, c'est l'ouverture circonstancielle à d'autres formes et d'autres genres qu'on soulignerait : la concurrence avec Eugène Sue depuis le triomphe des Mystères de Paris en 1842-1843 ; un traitement peut-être plus direct de la sexualité (avec l'érotomanie de Hulot, et l'aspect plus ou moins homosexuel des couples Lucien-Herrera, Bette-Valérie, Pons-Schmucke); la tentation du théâtre enfin ${ }^{2}$, peut-être visible dans les dialogues envahissants. On imagine alors un Cousin Pons expérimental, cherchant de nouvelles voies poétiques et thématiques; on rêve même au Balzac nouveau qui s'élaborait dans ce Cousin Pons, à un Balzac encore embryonnaire.

Lectures contrastées, que l'on peut certes faire converger: on peut s'émerveiller de la constance d'un projet (b) qui, dans une impasse existentielle et historique (a), ne néglige aucun des moyens d'expressions à sa portée (c). On peut aussi procéder par répartition : par exemple, épuisement dans le ton (la noirceur sarcastique), approfondissement dans les thèmes (le règne de l'argent), renouvellement dans la construction du récit (le récit feuilletonnant). Mais, aussi synthétique qu'elle soit, une lecture du Cousin Pons privilégiera tout de même une dominante, une «couleur » du texte. Je défendrai pour ma part une interprétation en termes de "renouvellement ». C'est un résultat de l'analyse exposée plus loin, mais c'est aussi un parti pris : en effet, parler de renouvellement, c'est d'une part résister à la lecture téléologique de l'œuvre (la mort de Balzac en 1850 «assombrissant » rétrospectivement l'année 1847) et d'autre part s'efforcer de repérer la singularité du Cousin Pons (ne pas retrouver La Comédie bumaine dans Le Cousin Pons, mais situer Le Cousin Pons par rapport à La Comédie humaine).

Où situer ce renouvellement? À la lecture, domine pour moi l'impression d'un rapetissement de l'univers balzacien, sur tous les plans. C'est à travers cette catégorie du " petit» que j'essaierai d'observer dans le Cousin Pons une modulation nouvelle de l'esthétique de Balzac - modulation qui se situe certes dans le prolongement de son projet d'ensemble, et qui ne va pas, on s'en doute, sans

\footnotetext{
${ }^{1}$ La manie accumulative de Gobseck, la vie anachronique du Colonel Chabert, la solitude persécutée de Pierrette et du Curé de Tours, les rêveries artistiques et musicales de Massimila Doni ou de Gambara, etc.

2 Signalée par Stéphane Vachon dans Le Dernier Balzac, Du Lérot, 2001.
} 
une nuance de pessimisme : on retrouvera l'approfondissement et l'épuisement. L'intérêt pour les " petites choses » n'est pas nouveau chez $\mathrm{Balzac}^{3}$; je verrai dans ce romanesque du «petit» une signification sociale et politique, la représentation des « petites choses » allant de pair avec celle des «petites gens ».

C'est à vrai dire le principe du roman balzacien (et du roman "réaliste" du début du XIX ${ }^{\mathrm{e}} \mathrm{s}$.) que de proposer ce changement d'échelle, ce déplacement du grand vers le petit: dans les termes d'Auerbach, c'est la représentation sérieuse de sujets traditionnellement bas. Chez Balzac, la représentation de ce monde bourgeois moderne (sur le plan social) se traduit par un projet d'histoire des mœurs privées, et aboutit à une mise en avant des détails (sur le plan poétique). Le détail balzacien peut être un détail significatif, comme la «cravate blanche à bouts brodés » de Lucien de Rubempré qui signale son identité provinciale ${ }^{4}$; ou un événement minuscule et trivial, comme le duel d'Eugénie et de son père autour du sucrier ${ }^{5}$, ou les pantoufles de l'abbé Birotteau ${ }^{6}$. Dans Le Cousin Pons, on peut évoquer le détail de la puanteur de l'appartement de Fraisier qui fait comprendre le célibat de Fraisier (p. 231), ou le grand effet produit sur Gaudissart par une petite cause :

«Ce féroce parvenu fut touché de cette noblesse et de cette reconnaissance pour une chose de rien aux yeux du monde [avoir suivi le convoi], et qui, aux yeux de cet agneau divin, pesait, comme le verre d'eau de Bossuet, plus que les victoires des conquérants » (p. 373)

Cette image du verre d'eau de Bossuet était déjà utilisée dans La Peau de chagrin ${ }^{7}$ et revient dans L'Envers de l'bistoire contemporaine.

Ainsi le monde bourgeois de la «vie privée» va de pair chez Balzac avec la poétique du détail. L'hypothèse proposée ici est qu'un « rapetissement » à la fois social et esthétique se produit dans Le Cousin Pons : le déplacement du personnel romanesque vers les «petites gens » (petits bourgeois et classes populaires) va de pair avec un rôle nouveau des " petites choses».

Ce rapetissement de l'univers balzacien (I), dont la collection de Pons offre un modèle en proposant une valeur du «petit » (II), va de pair avec un discours social et politique, qui permettra de mettre en perspective certaines interprétations du roman. On propose en ce sens une interprétation politique de ce rôle du « petit » dans Le Cousin Pons. (III)

\section{Un rapetissement de l'univers balzacien}

\section{Rapetissement social}

Le « dernier Balzac » cherche d'abord à faire évoluer son personnel romanesque, quant aux catégories sociales représentées. La préface de Splendeurs et misères des courtisanes est à cet égard éclairante :

L'aplatissement, l'effacement de nos moeurs va croissant. Il y a dix ans, l'auteur de ce livre écrivait qu'il n'y avait plus que des nuances ; mais aujourd'hui, les nuances disparaissent. Aussi (...) n'y a-til plus de mœurs tranchées et de comique possible que chez les voleurs, chez les filles, et chez les forçats, il n’y a plus d'énergie que dans les êtres séparés de la société?.

\footnotetext{
${ }^{3}$ Je renvoie sur ce point à l'article de Kathia Huynh sur le « romanesque du rien » (Le Cousin Pons de Balzac, dir. A. Déruelle, PUR, coll. «Concours », 2018) à entendre comme un romanesque des petits riens (et non d'un « livre sur rien » ou d'un roman du Néant).

${ }^{4}$ Illusions perdues, V, p. 269-270 (« le monde des superfluités nécessaires lui apparut »).

${ }^{5}$ Eugénie Grandet, III, p. 1091 («Certes, la Parisienne qui, pour faciliter la fuite de son amant, soutient de ses faibles bras une échelle de soie, ne montre pas plus de courage que n’en déployait Eugénie en remettant le sucre sur la table. »).

${ }^{6}$ Le Curé de Tours, p. 199 (« les quatre circonstances capitales de la porte fermée, des pantoufles oubliées, du manque de feu, du bougeoir porté chez lui »).

${ }^{7}$ La Peau de Chagrin, X, p. 163.

${ }^{8}$ L'Envers de l'Histoire contemporaine, VIII, p. 360.

${ }^{9}$ Préface de Splendeurs et misères, 1844, VI, p. 425.
} 
Ici ce sont les prostituées et les criminels qui, par leur position aux marges de la société, permettent de retrouver une dynamique romanesque, en opposant la marge à la norme. C'est une des ressources possibles dans une situation historique plus générale, marquée par une indifférenciation accrue entre les individus. De cette situation, l'incipit de Splendeurs et misères est un emblème, avec la déambulation in medias res d'un beau jeune homme anonyme, livré au déchiffrement indiciaire des « flâneurs émérites " ${ }^{10}$, confrontés au brouillage moderne des identités : Suivant une loi fatale de notre époque, il existait peu de différence, soit physique, soit morale, entre le plus distingué, le mieux élevé des fils d'un duc et pair, et ce charmant garçon que naguère la misère étreignait de ses mains de fer au milieu de Paris ${ }^{11}$.

Dans Le Cousin Pons, Balzac explore une autre ressource sociale, du côté des franges inférieures de la bourgeoisie et du « peuple » : employés, commerçants, et autres petites professions de Paris. On pourrait ainsi confronter une veine «criminelle " (Splendeurs et misères, la fin de La Cousine Bette) et une veine " petite-bourgeoise » ou "populaire» (Les Petits Bourgeois, Les Comédiens sans le savoir, Les Employés). Dans Le Cousin Pons, Balzac s'attache à répertorier une foule de "professions infimes » (99) (la profession étant un principe important de classement du monde social hérité de la littérature panoramique) : concierge, chef d'orchestre de théâtre, caissier de théâtre, commis aux monuments funéraires, loueuse de chaises à l'église, voyante, etc. Si «à Paris, chaque profession a ses Oméga » (p. 227), Le Cousin Pons se définit par ce parti pris de représenter les Oméga (l’homme de loi Fraisier, le médecin de quartier Poulain, le ferrailleur Rémonencq, etc.). On reconnaît l'impact des Mystères de Paris : et la rue de Normandie où habite Pons n'est pas sans évoquer l'immeuble de la rue Vieille-du-Temple, où Rodophe de Gerolstein rencontre les concierges M. et Mme Pipelet, la voyante Mme Burette, le médecin malhonnête César Bradamanti (alias l'hypocrite abbé Polidori, au centre d'un complot ténébreux), la famille pauvre et honnête des Morel, où l'on aperçoit facilement des modèles du couple Cibot, de Mme Fontaine, de Poulain et de la famille Topinard.

Les sommes d'argent extrêmement modiques, avec des comptes précis (ceux du ménage de Schmucke, p. 100-101, ou l'emploi des 12 sous par jours des Rémonencq, p. 162), rappellent aussi la volonté de Sue de renseigner sur le budget modique de la grisette Rigolette. Même choix aussi d'un ancrage dans un quartier populaire (le Marais du Cousin Pons, le quartier des Tuileries de La Cousine Bette, la rue de Langlade de Splendeurs et misères, émulent l'île de la Cité des Mystères de Paris). Cette veine «populaire» du Cousin Pons est une variante de la veine «criminelle» de Splendeurs et misères des courtisanes. On pourra ainsi rapprocher les incipits des deux romans : même entrée in medias res, même énigme quant à l'identité d'un personnage, même regard des " connaisseurs en flânerie " (variante des "flâneurs émérites" de Splendeurs), et même échec à catégoriser le personnage avant que le narrateur ne le dévoile - évidemment dans une tonalité moins mélodramatique.

Dans le même ordre d'idées, on peut noter que Topinard est relégué à la fin du roman dans "le troisième dessous d'un théâtre des boulevards ». Rappelons-nous ce passage de Splendeurs et misères des courtisanes, consacré à l'argot des criminels :

Disons-le, peut-être à l'étonnement de beaucoup de gens, il n'est pas de langue plus énergique, plus colorée que celle de ce monde souterrain qui, depuis l'origine des empires à capitale, s'agite dans les caves, dans les sentines, dans le troisième-dessous des sociétés, pour emprunter à l'art dramatique une expression vive et saisissante. Le monde n'est-il pas un théâtre? Le Troisième-Dessous est la dernière cave pratiquée sous les planches de l'Opéra, pour en recéler les machines, les machinistes, la rampe, les apparitions, les diables bleus que vomit l'enfer, etc. ${ }^{12}$

L'expression dans Le Cousin Pons a son sens littéral, mais sa position (la dernière page du roman) lui laisse aussi son sens figuré, social, de " troisième-dessous des sociétés ». Ainsi les pauvres du Cousin Pons sont comparables aux criminels de Splendeurs. De même, le désespoir du « jeune avocat sans causes » et du «jeune médecin sans clients » sont « un poème de tristesse, sombre comme les secrets de la Conciergerie.» (219). Et remarquons « les chineurs, dont le comique est

\footnotetext{
10 Splendeurs et misères des courtisanes, VI, p. 430

11 Ibid., p. 431.

12 Ibid., p. 828.
} 
bien plus franc que celui des négociateurs » (164). Balzac suggère que les petites gens - Topinard, Fraisier, Poulain - peuvent relayer les personnages de feuilleton comme sujets du romanesque.

\section{Rapetissement des motifs}

Le rapetissement social se répercute - ou s'accompagne - d'un rapetissement thématique, en particulier spatial. La critique a souvent noté que l'espace du Cousin Pons est restreint et fermé : du quartier étroit de la rue de Normandie à la chambre de Pons, il y a un mouvement de rétrécissement de l'espace. Ajoutons simplement, pour en marquer le caractère social, que même lors de l'ouverture de cet espace à la mort de Pons, Schmucke trouve un abri dans la cité Bordin, ce « faubourg saint Antoine en miniature » (366) : la fin du Cousin Pons évoque ainsi, sous une forme miniaturisée, la fin de La Cousine Bette - qui amenait le lecteur horrifié dans un faubourg saint Antoine sordide après ce qui était déjà une impressionnante trajectoire de décadence.

Psychologiquement, Pons est lui-même une version amenuisée des grands passionnés balzaciens : notons que dans Le Cousin Pons, toutes les passions sont viables : le principe de plaisir se combine avec le principe de réalité, sans que le désir ne mène à la folie ou à la mort. La bricabracomanie est une dépense, qui amène Pons à «zéro dans la bourse » (70), mais elle ne déclenche pas le terrible mécanisme balzacien de la dette. Pons paie la satisfaction de sa gastrolâtrie en "monnaie de singe » (65); ce faux péché capital ("le péché des moines vertueux »(67) est d'ailleurs, par rapport à la luxure de Hulot, « la Recette dont celle-ci est la Dépense » (64). Paul Bourget indique l'importance pour Balzac de ce problème des «petits vices » - encore qu'il en donne une version peut-être exagérément tragique :

C'est un très petit vice en effet et pour lequel le moraliste ne trouve guère de sévérité, que celui d'aimer à trop bien dîner. Le cousin Pons n'en a pas d'autre. Il ne s'en défie pas. Il le cultive. Tous ses malheurs et ceux de son ami Schmucke dérivent de cette innocente concession à un goût d'abord plutôt ridicule, mais dont le despotisme finit par bouleverser toute une existence. ${ }^{13}$

Par rapport à Bette, que l'on a pu identifier avec la pulsion de mort elle-même ${ }^{14}$, Pons serait du côté d'Éros, le désir polymorphe, qui se transforme sans cesse et passe d'un objet à l'autre.

Sur ce plan des «passions », Pons rejoue en mineur d'autres figures balzaciennes, pourtant souvent déjà elles-mêmes dégradées : Goriot, le vieillard grotesque, parent pauvre de ses propres gendres, enterré au Père-Lachaise dans l'indifférence de sa famille (mais du moins était-il le « Christ de la paternité »); Chabert, l'homme-Empire anachronique (mais du moins était-il un militaire, incarnation de l'Empire hérö̈que) ; et surtout Lucien de Rubempré, qui était déjà une figure d'artiste dégradé par le capitalisme moderne ${ }^{15}$. La mort de Pons fait écho à celle de Lucien : la mention de son monument funéraire, "l'un des plus magnifiques ornements du Père-Lachaise » (336) fait allusion à l'avant-dernière phrase de Splendeurs et misères ${ }^{16}$. Lucien, avec toutes ses faiblesses, était encore illuminé d'une certaine grandeur : jeune, beau reçu chez les Nucingen et les Grandlieu, talentueux et véritablement artiste ; Pons est vieux, laid, reçu au mieux chez les Camusot et les Popinot, n'ayant que le "génie de l'admiration, de la compréhension » (60) c'est-à-dire le génie des «homme[s] ordinaire[s] »(60), et renonce à «l'originalité qui distingue les artistes » à cause de son «habitude de s'effacer» (91). Plus rien à voir avec l'excès romantique, ni mêle avec ces « jovialités mirobolantes» (58) qu’imaginent les bourgeois.

Le rétrécissement thématique et social touche donc aussi la personnalité artistique. Le fait que Pons soit collectionneur doit se lire par opposition avec tous les artistes de la Comédie bumaine: Lucien, Nathan, Frenhofer, Conti, etc. La collection, affaire d'amateurs et de connaisseurs, renvoie aussi à un art du « petit».

\footnotetext{
${ }_{13}$ Paul Bourget, «Balzac et Le Cousin Pons (1898), Nouvelles pages de critique et de doctrine, 1922, p. 50.

${ }^{14}$ Fredric Jameson, «La Cousine Bette and Allegorical Realism », PMLA, Vol. 86, n² (mars 1971), p. 241-254.

${ }^{15}$ Georg Lukács, «Illusions perdues », dans Balz̧ac et le réalisme français [1935], Paris, La Découverte, 1999.

${ }^{16}$ Cf. Aude Déruelle (communication sur la mort dans Pons dans journée d’études GIRB-GEB)
} 


\section{II. «Des petites bêtises!» : la collection de curiosités}

On pourrait développer le rôle de tous les « riens » auxquels Balzac donne de l'importance dans le roman (comme ces « mille riens » de l'habitude, p. 108). Mais une particularité de Pons est la thématisation de cette esthétique du détail dans la collection de Pons.

\section{Une collection de petites choses}

La collection apparait comme une des figures privilégiées du « petit» dans le texte. C'est le « lingot du bonheur » en "petite monnaie $»^{17}$; ce sont de «petites curiosités » (308), de "petites bêtises $»^{18}$, des "brimborions ${ }^{19}$, des "biblots» (206, 243, 257). En effet, la collection Pons rassemble beaucoup de petits objets : à côté des tableaux et des bronzes, qu'on peut imaginer imposants par la taille, il faut remarquer les miniatures et autres objets de petite taille, signalés à plusieurs reprises :

Le premier, Pons avait collectionné les tabatières et les miniatures (62)

des vitraux, des émaux, des miniatures, des tabatières en or et en argent (284)

mes tableaux, mes curiosités, mes tabatières, mes miniatures (286)

les plus grandes raretés du travail humain : les ivoires, les bronzes, les bois, les émaux, l'orfèvrerie, les porcelaines, etc. » (204)

Chaque carreau des deux croisées de la chambre du bonhomme était un vitrail suisse colorié (136)

elles furent surprises de la beauté de quelques bijoux étrusques et de la valeur réelle des tabatières. Elles s'extasiaient par complaisance en tenant à la main des bronzes florentins (135)

Parmi les tableaux, notons le «sublime petit tableau de Metzu» que la Cibot peut transporter sans difficulté ; mais aussi les « fleurs de Van Huysum, de David de Heim, les insectes d'Abraham Mignon » (135). L'éventail de Watteau est un emblème de cette prédilection pour le petit, auquel s'attachent plusieurs significations :

- l'idée d'une valeur marchande infiniment concentrée : c'est la dimension du précieux (cf. La

Paix du ménage, où Balzac rappelle que la «passion effrénée pour tout ce qui brillait » de l'Empire pour le diamant tient aussi à ce que le diamant est « le butin sous la forme la plus facile à transporter $\left.»^{20}\right)$. Les objets de Pons sont comparés à des diamants $(204,318)$

- La valeur peut donc échapper au regard inattentif : il faut avoir l'œil, être capable de percevoir l'imperceptible, l'éventail dans sa boîte et la boîte dans le meuble. "C'est des combats d'œil à œil » (89).

- De la part de Pons, c'est aussi un choix financier : les porcelaines sont moins chères que les meubles de Riesener (86). En même temps qu'il est du côté du précieux, le petit est du côté du sous-estimé.

Ce point nous renvoie à l'aspect social du petit. La cité Bordin, ce « faubourg saint-Antoine en miniature », est d'ailleurs lié à ces petits objets artistiques :

On y fait des meubles, on y cisèle les cuivres, on y coud des costumes pour les théâtres, on y travaille le verre, on y peint les porcelaines, on y fabrique enfin toutes les fantaisies et les variétés de l'article Paris.

\footnotetext{
${ }^{17} 63$; l'image de la «monnaie d'une femme » se retrouve p. 67

18 selon Mme Camusot (81), Cécile Camusot (120) et Schmucke (104)

${ }^{19}$ selon Cécile, p. 137 ; mais aussi selon Schmucke, p. 190 et p. 281 ("primporions»); notons aussi l'emploi de ce terme p. 229.

${ }^{20}$ La Paix du ménage, II, p. 96.
} 
Ainsi la collection est placée du côté de la fragmentation, mais indique aussi une valeur intrinsèque du petit. Valeur esthétique, valeur marchande, valeur historique aussi - ces bibelots ne sont-ils pas « les débris du dix-septième et du dix-huitième siècle » (61)?

\section{Justification du petit par le grand: l'archéologie et les curiosités}

C'est le président Camusot qui est chargé - à contre-emploi - de défendre la collection de Pons, en s'offusquant du terme « petite bêtise »employé par sa femme et sa fille (p. 83, p. 120). Il s'y emploie en recourant au modèle de l'archéologie :

— Des petites bêtises ! s'écria le président. (...) Ces petites bêtises-là, ma chère enfant, sont souvent les seuls témoignages qui nous restent de civilisations disparues. Un pot étrusque, un collier, qui valent quelquefois, l'un quarante, l'autre cinquante mille francs, sont des petites bêtises qui nous révèlent la perfection des arts au temps du siège de Troie, en nous démontrant que les Étrusques étaient des Troyens réfugiés en Italie.

Les petites choses sont ici défendues pour leur valeur de signes et plus précisément d'indices, dont une interprétation permet de dégager un sens plus vaste que leur existence ${ }^{21}$. Du petit sort le grand.

Effectivement la collection a une valeur de synecdoque d'une civilisation (comme celle de l'Antiquaire de la Peau de Chagrin) : l'éventail indique Watteau et Mme de Pompadour ; les quatre tableaux principaux (204-205) forment une synthèse de la peinture, comme l'a indiqué Michel Butor :

Sebastiano del Piombo n'est ni le Titien, ni Giorgione, mais Balzac montre très bien la position de carrefour qu'il a dans l'art italien. Fra Bartolomeo est un second pour Raphaël. Hobbema apporte l'art hollandais. Il n'est ni Rembrandt, ni même, pour le paysage, Ruysdaël. Mais il transmet une tradition de haute bourgeoisie tandis que Sebastiano transmet la noblesse vénitienne, fra Bartolomeo l'Église romaine. Quant à Dürer il apporte l'art allemand ${ }^{22}$

On pourrait aussi dire, toujours en voyant en ces quatre tableaux une synthèse de l'Art tout entier plutôt qu'une série d'œuvres singulières valant pour elles-mêmes : art italien, avec le portrait (portrait de Sebastiano del Piombo) et l'art sacré (la Sainte Famille à la Raphaël) ; art du Nord, avec le paysage (Hobbéma) et le portrait (Dürer).

Quoi qu'il en soit, la collection de Pons est réévaluée comme une sorte d'arche d'une civilisation disparue :

La réunion des connaissances qu'exigent ces petites bêtises, Cécile, reprit-il, est une science qui s'appelle l'archéologie. L'archéologie comprend l'architecture, la sculpture, la peinture, l'orfèvrerie, la céramique, l'ébénisterie, art tout moderne, les dentelles, les tapisseries, enfin toutes les créations du travail humain. (p. 120)

Camusot, décidément plus profond qu'il n'en a l'air, envisage une archéologie encyclopédique qui englobe même "l'ébénisterie, art tout moderne »: une archéologie de la modernité, donc, ce qui est tout à fait le projet de Balzac.

Notons alors que cette valorisation « archéologique » du petit se fait en marge de la beauté. À côté d'une valeur esthétique (que représenteraient surtout, dans la collection, les tableaux), il y a une valeur du curieux (de l'étrange, de l'insolite, du remarquable). Ces curiosités sont, me semble-til, la spécificité de la collection de Pons par rapport à celle de Magus :

— Oh! milord, dit modestement le comte Popinot, en fait de tableaux, personne, je ne dirai pas à Paris, mais en Europe, ne peut se flatter de rivaliser avec un inconnu, un Juif nommé Élie Magus, vieillard maniaque, le chef des tableaumanes. Il a réuni cent et quelques tableaux qui sont à décourager les amateurs d'entreprendre des collections. La France devrait sacrifier sept à huit millions et acquérir cette galerie à la mort de ce richard... Quant aux curiosités, ma collection est assez belle pour qu'on en parle... [c'est moi qui souligne]

C'est d'autant plus vrai que Magus a dépouillé la collection de ses quatre plus beaux tableaux, Rémonencq de quatre autres, et la Cibot d'un neuvième. La division proposée ici entre

\footnotetext{
${ }^{21}$ Sur cette poétique balzacienne du signe, voir Boris Lyon-Caen, Balzac et la comédie des signes, P. U. Vincennes, 2006.

${ }^{22}$ Michel Butor, Paris à vol d'archange. Improvisations sur Balzac, vol. II, p. 292.
} 
tableaux et « curiosités » semble donner à ces dernières un statut spécifique ${ }^{23}$, une valeur propre et peut-être en un sens plus haute que la beauté des tableaux. En témoigne l'emploi métalittéraire de ce terme : le roman se donne comme une galerie de curiosités, à l'instar du Cabinet des antiques (1838). Pons est présenté dès la première page comme une de ces « hautes curiosités vivantes ", un antique à «valeur archéologique » (p. 53), un « ensemble de petites choses qui voulait l'attention analytique des connaisseurs en flânerie » (54).

Même apologie du petit comme signe du grand dans le traité d'occultisme, que JacquesDavid Ebguy interprète à juste titre comme un passage métalittéraire ${ }^{24}$ :

Les moindres accidents, les plus futiles, y sont subordonnés. Donc les grandes choses, les grands desseins, les grandes pensées s'y reflètent nécessairement dans les plus petites actions

On retrouve ainsi dans la voyance «la faculté de déduction qui fit Cuvier si grand » (176) que l'auteur de La Peau de Chagrin se donnait pour modèle.

Balzac considère les sciences occultes comme un principe général à l'œuvre dans des domaines disparates, mais qui ont en commun de trouver une signification dans le minuscule, l'imperceptible :

- «La crânologie, la physiognomonie, la névrologie » (176)

- La photographie, comme captation d'un objet dans l'atmosphère (173) - ce qui n'est pas sans évoquer les simulacres de Lucrèce, faits d'atomes ténus;

- La connaissance sociale : «La plupart des observateurs de la nature sociale et parisienne peuvent dire la profession d'un passant en le voyant venir. » (174) ; c'est le procédé de la littérature panoramique.

Il y a pour Balzac continuité entre le corps et l'esprit, entre le perceptible et l'imperceptible.

\section{Sensibilité et vulnérabilité}

Cela dit, la collection de Pons est une modulation plus modeste de ce projet. Il n'est pas anodin que le thème soit défendu par un imbécile, Camusot, qui y met trop d'emphase :

Tel était le genre de plaisanterie du gros petit président, il procédait avec sa femme et sa fille par de lourdes ironies.

Il y faudrait peut-être plus de finesse, et moins de lourdeur démonstrative.

S'il est vrai que la comparaison de la peinture à un paraphe (89), que l'insistance sur la signature (des porcelaines, 86 ; de l'éventail, 89), assimilent la collection à une écriture (livre ou bibliothèque), elles signalent surtout la finesse de Pons. C'est-à-dire son attention au détail, sa capacité à percevoir un grain plus fin de la réalité, comme le « tout petit rubis » (90) qu’il a décrassé. Notons que cette attention au détail est concentrée sur la signature (plus que sur l'écriture d'un sens), c'est-à-dire à la fois sur l'inscription d'un sujet dans son œuvre, et sur l'attribution d'une valeur à cette œuvre (celle-ci découlant de celle-là). C'est la même finesse qu'on retrouve dans sa gourmandise, il est "gueule fine»(63). Cette sensibilité esthétique est apparentée à sa sensibilité affective : sa capacité à trouver « une signifiance psychique aux riens de la création » (70), et bien sûr cette sensibilité organique au moindre grain de sable, là où la société contemporaine n'a plus qu'un rapport mécanique au monde, celui de la locomotive qui brûle les grains de sable sur ses rails (72).

\footnotetext{
${ }^{23}$ Le terme revient 35 fois dans le roman, avec ses diverses acceptions subjectives et objectives. Notons tout particulièrement, à propos de l'occultisme : «un sentiment inné, l'un des plus forts de notre nature, la Curiosité » (p. 172).

${ }^{24}$ Jacques-David Ebguy, « Un arrêt au passage. Le Cousin Pons, roman de la spectralité », p. 211-238 dans Relire Le Cousin Pons, dir. É. Reverzy et P. Glaudes, Classiques Garnier, 2018.
} 


\section{Une représentation des rapports sociaux après 1830}

\section{Ceux qui ne sont rien}

Notons que cette métaphore à trois termes (le grain de sable, l'organisme et le chemin de fer est une matrice (p. 72) qui se décline selon deux emplois distincts dans le roman : d'un côté, en ne conservant que le grain de sable et l'organisme, on a les images de la sensibilité que nous venons de commenter (ainsi p. 90 : «Ce gravier un peu trop gros lui roula pendant quelque temps dans le cœur »); de l'autre, en conservant la locomotive et le grain de sable, on obtient les images du personnage broyé par la société, mettant en évidence la vulnérabilité du personnage, qui se sent à son tour rapetissé :

« cette société qui roule sur moi, comme un tombereau sur un œuf » (166)

L'image désigne alors les victimes de la société, et de son mouvement, l'Histoire. Les lecteurs du Père Goriot reconnaissent l'image de l'incipit :

Le char de la civilisation, semblable à celui de l'idole de Jaggernaut, à peine retardé par un cœur moins facile à broyer que les autres et qui enraye sa roue, l'a brisé bientôt et continue sa marche glorieuse. Ainsi ferez-vous, vous qui tenez ce livre d'une main blanche, vous qui vous enfoncez dans un moelleux fauteuil en vous disant : Peut-être ceci va-t-il m'amuser. ${ }^{25}$

Cette crainte est en fait dans Le Cousin Pons le lot commun des petites gens, et Fraisier sait bien l'exploiter pour effrayer la Cibot :

Mais le bonhomme a ses héritiers en horreur ; il me répète que ces gens-là (...) l'ont écrasé comme un œuf qui se trouverait sous un tombereau.

— Voulez-vous être broyée ainsi ? ... (236)

Autre variante de l'image du gravier : les gens du peuple comparés à des « cailloux roulés dans les torrents de la misère » dans une métaphore filée (177).

La métaphore du grain de sable sert d'embrayeur entre le discours psychologique (la sensibilité artistique et affective de Pons et Schmucke) et le discours politique (la vulnérabilité des petites gens). Sur ce plan politique, le thème de la petitesse, de la quantité imperceptible, prend tout son sens.

Et Balzac considère son écriture comme capable de dire ces imperceptibles quantités sociales, ces « curiosités » qu'il faut décrire en détail. Il peut sans doute faire sienne cette réplique de Fraisier à la Sauvage qui s'intéresse peu à Topinard :

- Vous voyez ce petit misérable !... C'est une espèce d'honnête homme qui veut fourrer son nez dans les affaires de monsieur Schmucke...

- Qui est-ce ? demanda Fraisier.

- Oh! un rien du tout...

- Il n'y a pas de rien du tout, en affaires..

\section{Leçons d'homéopathie criminelle}

Je voudrais m'attarder sur le meurtre imaginatif de Cibot par Rémonencq, au moyen d'oxyde de cuivre ajouté en quantité imperceptible dans la tisane de son rival. C'est une forme originale de l'idée balzacienne des petites causes produisant de grands effets, bien connue, dans la préface des Études de mours par Félix Davin ou dans La Vieille Fille:

Ces petites choses décident de la fortune des hommes, comme de celle des empires. (...) personne n'en profite pour ne rien négliger dans les petits faits de sa vie ${ }^{26}$.

Cette fois l'idée est poussée plus loin, jusqu'à l'idée très romanesque d'une « imperceptible dose de poison », selon le génial principe de l'« homéopathie criminelle » (294)

Cette légère addition de cuivre chargé de son oxyde, communément appelé vert-de-gris, introduisit secrètement un principe délétère dans la tisane bienfaisante, mais en proportions homéopathiques, ce qui fit des ravages incalculables. (294)

Faut-il y voir une ironie vis-à-vis de la tirade favorable à l'homéopathie dans Les Mystères de Paris (IX partie, ch. 5) ? Pourquoi pas, mais c'est surtout bien sûr un doublet symbolique du « crime moral »

${ }^{25}$ Le Père Goriot, III, p. 50.
${ }^{26}$ La Vieille Fille, IV, p. 906. 
dont Pons est simultanément victime, le poison microscopique étant le pendant matériel du fameux « grain de sable » moral. C'est d'ailleurs un principe semblable qui agit dans le meurtre suivant, où joue la différence imperceptible (ou plutôt non perçue) entre le verre d'eau - mais il ne s'agit plus de celui de Bossuet cette fois-ci ! - et le verre de vitriol.

La rondelle utilisée par Rémonencq est d'ailleurs "ronde comme une pièce de cent sous », cette même pièce qui « est tapie dans toutes les consciences » et « roule dans toutes les phrases » (217) depuis 1830 ! C'est toute la vie sociale du régime de Juillet qui est métaphorisée dans ces meurtres finaux comme un empoisonnement moral.

L'humour noir de la situation tient à ce que Poulain est incapable, malgré ses soupçons, de déceler le meurtre :

Le docteur Poulain se creusa la tête en apercevant l'effet de cette décoction, car il était assez savant pour reconnaître l'action d'un agent destructeur. Il emporta la tisane, à l'insu de tout le monde, et il en opéra l'analyse lui-même ; mais il n'y trouva rien. Le hasard voulut que, ce jour-là, Rémonencq, effrayé de ses œuvres, n'eût pas mis sa fatale rondelle.

Ce sont les limites de l'« analyse » des « connaisseurs » dont l'incipit faisait un modèle.

Le docteur Poulain s'en tira vis-à-vis de lui-même et de la science, en supposant que, par suite d'une vie sédentaire, dans une loge humide, le sang de ce tailleur accroupi sur une table, devant cette fenêtre grillagée, avait pu se décomposer, faute d'exercice, et surtout à la perpétuelle aspiration des émanations d'un ruisseau fétide. (...)

- Quelle est donc la maladie de mon pauvre Cibot ? avait demandé la portière au docteur Poulain. — Ma chère madame Cibot, répondit le docteur, il meurt de la maladie des portiers... son étiolement général annonce une incurable viciation du sang. (294-295)

Poulain, au lieu de reconnaitre l'action « moléculaire » du poison de Rémonencq, à l'échelle des individus, élabore tant bien que mal une interprétation «molaire $»^{27}$, grossière et factice ( la maladie des portiers »), et se laisse prendre à une généralisation misérabiliste, qui lui fait manquer l'idée fixe de Rémonencq.

Une assez grande quantité de crimes échappent à la vengeance de la société, c'est en général ceux qui se commettent, comme celui-ci, sans les preuves effrayantes d'une violence quelconque : (...) mais surtout quand le meurtre est sans intérêt apparent, et commis dans les classes inférieures. Le crime est toujours dénoncé par son avant-garde, par des haines, par des cupidités visibles dont sont instruits les gens aux yeux de qui l'on vit.

Poulain, manquant la présence active, entre les individus, de relations directes de violence, est contraint d'imaginer la «maladie des portiers », c'est-à-dire un effet imaginaire du milieu.

\section{Pour un Balzac moléculaire...}

L'image de l'action minuscule et dévastatrice de la rondelle de cuivre, dont la forme est si semblable à celle de l'omniprésente "pièce de cent sous ", indique que la modernité n'est pas seulement le règne des grands capitaux, de l'opposition «molaire ", à grande échelle, entre les bourgeois et les prolétaires, entre le clan Camusot et le couple Pons-Schmucke; c'est l'invasion de toutes les relations sociales par l'argent, même sous la forme de sommes dérisoires, qui constitue la société comme une course des cupidités.

Il s'agit en somme de nuancer l'interprétation du roman en termes d'opposition structurale entre classes sociales, qui fait fond sur l'antagonisme initial entre les Camusot et Pons : dans le conflit entre la famille et le parent pauvre, le roman mettrait en scène l'opposition entre la bourgeoisie et la culture ${ }^{28}$ (c'est-à-dire le résidu vivant de l'Ancien Régime), ou entre la bourgeoisie et un prolétariat qui ne se connaît pas encore ${ }^{29}$.

\footnotetext{
${ }^{27}$ J'emprunte l'opposition molaire/moléculaire, assez librement, à Gilles Deleuze et Félix Guattari (Mille Plateaux, Minuit, 1980). Le propos est ici aussi d'opposer le fonctionnement du niveau « microscopique » à l'organisation structurale du niveau « macroscopique ».

${ }^{28}$ Pierre Barbéris, Mythes balz̧aciens, Armand Colin, 1972.

${ }^{29}$ Dans Mythes balzaciens, Pierre Barbéris agence son parcours de telle sorte que la victoire de la bourgeoisie (Le Cousin Pons, La Cousine Bette) prépare l'émergence encore confuse du prolétariat (Les Paysans).
} 
D'autres lectures ont montré la fragilité de cette opposition structurale: en Pons le grotesque côtoie le sublime ${ }^{30}$, la valeur marchande contamine la jouissance de l'art pur ${ }^{31}$. L'univers du Cousin Pons serait de part en part ambigu, brouillé ; l'idéal y serait absent, hormis sous la forme de vestige (la collection, la musique, la poésie). C'est une lecture flaubertienne ${ }^{32}$ : dans le monde bourgeois "d'après la coupure »" l'idéal est illusoire, la pensée et la parole sont des idées reçues, l'artiste n'a plus de position possible. Cette «flaubertisation » de Balzac est-elle tenable ? Il n'est pas évident qu'on puisse articuler les procédés balzaciens et parfois feuilletonnesques du Cousin Pons avec l'esthétique encore à venir de Madame Bovary ${ }^{34}$.

Ces lectures partent toutes du contraste, certes posé comme matriciel dans le roman, entre Pons et les Camusot - soit pour y voir un antagonisme historique, soit pour dissoudre l'opposition apparente en indifférenciation profonde. Contraste «molaire ", à grande échelle, que je voudrais compléter par une lecture plus « moléculaire » : avec les " petites gens », on remet au centre de la lecture des personnages "secondaires $»^{35}$; ce sont eux, après tout, qui ont transformé la nouvelle en $\operatorname{roman}^{36}$.

Pour combler l'écart entre Pons et les Camusot, pour que l'héritage de Pons arrive jusqu'aux Camusot, le roman mobilise en effet une armée de personnages secondaires et intermédiaires ${ }^{37}$ : Rémonencq, Poulain, Mme Cibot, Trognon, Gaudissard, Héloïse Brisetout, etc. Les petites différences monnaient ainsi la grande opposition. L'argent s'infiltre dans la texture fine des rapports sociaux, non pas selon l'opposition entre ceux qui ont et ceux qui n'ont pas, mais selon un schéma de réseau qui, par entrelacement feuilletonnesque, met en scène les petites alliances et les petits bénéfices.

Le modèle de l'intrigue est alors une chaîne d'alliances et de subordinations, où chacun recrute le suivant en se plaçant sous ses ordres :

- Rémonencq a besoin de la Cibot pour pénétrer chez Pons (164-165); elle le met au pas (171).

\footnotetext{
${ }^{30}$ Ruth Amossy, «L'esthétique du grotesque dans Le Cousin Pons», Balzac et Les Parents pauvres, dir. Françoise RossumGuyon et Michiel van Brederode, CDU-SEDES, 1981.

31 Franc Schuerewegen, "Muséum ou Croutéum? Pons, Bouvard, Pécuchet et la collection », Romantisme, 1987, no 17, vol. 55 (le brouillage de l'opposition structurante du roman est à rapprocher des lectures déconstructionnistes de Balzac contre Balzac. Les Cartes du lecteur) ; Pierre-Marc de Biasi, "Système et déviance de la collection à l'époque romantique », Romantisme, 1980, nº 27.

${ }^{32}$ Franc Schuerewegen construit son interprétation par un couplage du Cousin Pons avec Bouvard et Pécuchet; P.-M. de Biasi est spécialiste de Flaubert. L'article de Ruth Amossy lorgne plutôt du côté de Hugo.

${ }^{33}$ F. Schuerewegen, art. cit., p. 53. La « coupure» désigne habituellement 1848, le passage d'un premier XIX ${ }^{\mathrm{e}}$ siècle héroïque et romantique, animé par une Histoire ouverte vers l'avenir, à un second $\mathrm{XIX}^{\mathrm{e}}$ siècle désillusionné, où l'écrivain se met en retrait d'une Histoire désormais fermée. Pierre Barbéris signale lui aussi, dans sa perspective, cette appartenance du Cousin Pons à l'époque «flaubertienne »: « À partir de là tout va peut-être repartir, et par les moyens non plus de l'Histoire mais de l'art et de la littérature. On entre dans la seconde moitié du siècle. » (op. cit., p. 257)

34 Cette «flaubertisation » peut faire passer Balzac pour un Flaubert inachevé qui ne disposerait pas encore de ses procédés formels. F. Schuerewegen suggère que l'ironie balzacienne passe plutôt par le brouillage actantiel, et s'efforce parallèlement de repérer des procédés d'ironie flaubertienne dans le Cousin Pons: idées reçues, discours indirect libre, citations et répétitions (art. cit.).

35 Secondaires, du moins, dans ces interprétations très globales. Barbéris en particulier, dans son chapitre certes extrêmement concis (op. cit., p. 255-263), ne cite Schmucke qu'une fois au détour d'une phrase, et ne dit rien de Fraisier, de madame Cibot et de Rémonencq. Seul Magus surnage et même domine, dans la mesure où il incarne tout ce qui échappe finalement à la bourgeoisie.

${ }^{36}$ Voir l'analyse de la genèse du roman par Anne-Marie Meininger dans son édition (Balzac, Le Cousin Pons, Classiques Garnier, 1974, p. XII-LXIX).

${ }^{37}$ Fredric Jameson note à propos de la disparition progressive de la cousine Bette : «If the characteristically Balzacian plot is a duel between two wills, this gradual complication, in which little by little mediators interpose themselves between the two principal enemies, is also profoundly characteristic» ("si l'intrigue balzacienne typique est un duel entre deux volontés, cette complication graduelle, où petit à petit des médiateurs s'interposent entre les deux ennemis principaux, est aussi profondément typique ») (F. Jameson, art. cit., p. 245).
} 
- Madame Cibot fait appel à Poulain pour influencer le malade (222) ; « effrayé de perdre son honnêteté pour si peu de chose » (223), Poulain la fait entrer dans un jeu plus vaste en lui recommandant Fraisier.

- Madame Cibot fait appel à Fraisier, apparemment obéissant comme « un jeune soldat écoutant un vieux de la vieille » (232), mais il la domine rapidement (ch. XLVI-XLVII) : « je serait toute à vous » (244); plus tard, elle finira « matée » (320).

- Enfin, Fraisier a besoin des Camusot pour satsifaire son ambition; aussi se met-il franchement à leur service (ch. LII-LIII) - ce qui ne l'empêche pas de se munir de pièces éventuellement utiles contre eux en guise de garantie (320).

On pourrait représenter cela comme une série de schémas actantiels où chaque sujet (Rémonencq, puis madame Cibot, puis Fraisier) recrute un adjuvant dont l'aide ne peut être obtenue qu'en intervertissant les rôles (madame Cibot ne peut obtenir l'aide de Fraisier qu'à la condition qu'il prenne le contrôle des opérations, de sorte qu'elle est rétrogradée au rang d'adjuvant de son adjuvant devenu sujet).

Chacun, le long du fil inévitable qui relie le parent pauvre à la famille, prélève une part des profits. Il s'agit pour chacun d'eux de "faire sa pelote » ${ }^{38}$ c'est-à-dire d'accumuler de "légers bénéfices » (162) sur un mourant dont le destin est déjà joué.

Parallèlement à cette intrigue d'héritage, qui se construit comme une chaîne de vassalités âprement négociées, se met en place une deuxième chaîne du même type autour de la vente des tableaux, avec Rémonencq, madame Cibot et Élie Magus. En effet, l'organisation de ce réseau n'est pas purement linéaire, malgré la mise en place de « chaînes » entre les personnages : Fraisier ayant dissipé l'illusion de madame Cibot et lui ayant montré qu'elle ne pouvait être bénéficiaire principale du testament de Pons (234-239), celle-ci commence un second programme narratif, consistant à dépouiller Pons de quelques tableaux («faisons ma pelote », 245). L'intrigue bifurque. Pour ce faire, elle doit constituer une deuxième chaîne (avec Rémonencq, et Magus comme bénéficiaire final, d'autant plus qu'il rachète les tableaux de Rémonencq (282) - notons que madame Cibot, malgré tous ses efforts, n'est jamais que dans une position d'intermédiaire, rémunératrice financièrement mais non seconde symboliquement). Les deux programmes narratifs, menés par Fraisier et par la Cibot, se concurrencent (259) au risque de se nuire: la vente des tableaux (ch. LV), avouée par Schmucke (ch. LVII), donne à Pons l'idée d'un piège (ch. LIX-LX) qui met en péril la captation d'héritage. Les deux programmes s'entrelacent et finalement coexistent pacifiquement (356); d'ailleurs à un autre point de vue, la vente des tableaux, en précipitant sans doute la mort de Pons, était nécessaire pour la captation d'héritage.

Pourquoi entrer dans ce niveau de détail ? Pour montrer que l'enjeu narratif du roman, à partir du moment où Pons est malade, n'est pas tant dans l'opposition entre le parent pauvre Pons et la famille bourgeoise des Camusot, que dans la genèse d'une intrigue de prédation, dont les bénéficiaires sans scrupules sont les Camusot (ou plutôt les Popinot), mais dont les sujets sont les impitoyables «petites gens » du Marais.

Cela dessine un réseau fin et complexe de micropouvoirs ${ }^{39}$, plutôt qu'un grand contraste entre les puissants et les dépossédés. La médecine, dans son rôle de diagnostic et de conseil ; le droit, dans sa capacité à lier et à menacer ${ }^{40}$; le « soin » aussi, sous la forme des bavardages insidieux de la Cibot.

\footnotetext{
38 «Faire ma pelote! » s'indigne hypocritement madame Cibot (209), avant de conclure : « faisons ma pelote » (245). Fraisier le lui dit : « vous avez fait votre pelote, elle est dodue !» (356)

${ }^{39}$ On pourrait à cet égard prolonger la lecture foucaldienne proposée par Lucienne Frappier-Mazur, «Le discours du pouvoir dans Le Cousin Pons », Balzac et Les Parents pauvres, dir. Françoise Rossum-Guyon et Michiel van Brederode, CDU-SEDES, 1981.

${ }^{40}$ Le droit dans Le Cousin Pons a souvent un rôle d'intimidation : Fraisier effraie la Cibot à plusieurs reprises (234, 259, 319) ; il chasse Schmucke puis le dépouille par des manœuvres d’intimidation sans solidité légale (363, 376-377) Pons lui-même y a recours en imaginant le faux testament.
} 
C'est ce que Balzac met au centre de son intrigue : la toute-puissance de la pièce de cent sous, dans la mesure où, aux niveaux même les plus infimes de la société, tout un groupe social s'organise spontanément de manière prédatrice pour extorquer les sommes les plus minimes.

Cela concerne l'ensemble du tissu social, des plus pauvres aux plus riches. À un bout de la chaîne, Schmucke, pourtant réduit au plus extrême dénuement, est encore la proie des vendeurs de monuments funéraires et des mendiants :

Le bas clergé de l'Église, les pauvres, les croque-morts, les cochers, les fossoyeurs, ces natures spongieuses se retirent gonflées en se plongeant dans un corbillard. De l'église, où l'héritier à sa sortie fut assailli par une nuée de pauvres, aussitôt réprimée par le suisse, jusqu'au Père-Lachaise, le pauvre Schmucke alla comme les criminels allaient du Palais à la place de Grève. (349)

Ce sont «les pauvres » eux-mêmes, qui font ainsi régner la loi de la cupidité entre eux. À l'autre bout, n'oublions pas que les Camusot eux-mêmes sont à leur tour des « pauvres » :

la présidente se trouvait presque pauvre au milieu de la société de bourgeois parvenus où dînait Pons. (84)

Dire à un riche : «Vous êtes pauvre !» c'est dire à l'archevêque de Grenade que ses homélies ne valent rien. Madame la présidente était beaucoup trop orgueilleuse de la position de son mari, de la possession de la terre de Marville, et de ses invitations aux bals de la cour, pour ne pas être atteinte au vif par une semblable observation, surtout partant d'un misérable musicien vis-à-vis de qui elle se posait en bienfaitrice. (83)

— Je ne comprends rien au temps où nous vivons, répondit la présidente. À quoi cela sert-il donc d'avoir pour père un président à la Cour royale de Paris, et commandeur de la Légion-d'Honneur, pour grand-père un député millionnaire, un futur pair de France, le plus riche des marchands de soieries en gros ? (...)

— On ne veut que de l'argent aujourd'hui, répondit le cousin Pons, on n'a d'égards que pour les riches, et...

— Que serait-ce donc, s'écria la présidente, si le ciel m’avait laissé mon pauvre petit Charles ? ...

— Oh ! avec deux enfants, vous seriez pauvre ! reprit le cousin. C'est l'effet du partage égal des biens (91)

Voilà donc le récit d'un monde sous hégémonie bourgeoise : la mise en place d'alliances hétéroclites, mais organisées par une idée fixe uniforme d'ascension sociale, sur fond de frustration généralisée, et qui n'est pas exercée de haut en bas, mais uniformément, depuis la cupidité des riches jusqu'à la « jalousie dont sont dévorées les professions infimes à Paris » (99). 